\title{
Structural Stiffness Identification Based on the Extended Kalman Filter Research
}

\author{
Fenggang Wang, ${ }^{1,2}$ Xianzhang Ling, ${ }^{1,3}$ Xun Xu, ${ }^{1}$ and Feng Zhang ${ }^{1}$ \\ ${ }^{1}$ School of Civil Engineering, Harbin Institute of Technology, Harbin 150090, China \\ ${ }^{2}$ Northeast Forestry University, Harbin 150040, China \\ ${ }^{3}$ State Key Laboratory of Frozen Soil Engineering, Cold and Arid Regions Environmental and Engineering Research Institute, \\ CAS, Lanzhou 730000, China
}

Correspondence should be addressed to Fenggang Wang; wang_fenggang@163.com

Received 5 January 2014; Revised 24 March 2014; Accepted 27 March 2014; Published 21 May 2014

Academic Editor: Shuping He

Copyright (C) 2014 Fenggang Wang et al. This is an open access article distributed under the Creative Commons Attribution License, which permits unrestricted use, distribution, and reproduction in any medium, provided the original work is properly cited.

\begin{abstract}
For the response acquisition of the structure section measuring points, the method of identifying the structural stiffness parameters is developed by using the extended Kalman filter. The state equation of structural system parameter is a nonlinear equation. Dispersing the structural dynamic equation by using Newmark- $\beta$ method, the state transition matrix of discrete state equation is deduced and the solution of discrete state equation is simplified. The numerical simulation shows that the error of structural recognition doesnot exceed $5 \%$ when the noise level is $3 \%$. It meets the requirements of the error limit of the engineering structure, which indicates that the derivation described in this paper has the robustness for the structural stiffness recognition. Shear structure parameter identification examples illustrate its applicability, and the method can also be used to identify physical parameters of large structure.
\end{abstract}

\section{Introduction}

The dynamic response of structure which is developed in recent years is used to recognize the structural damage. This method is based on the structural dynamic parameter. The civil engineering structure will accumulate damage because of the collision, environment corrosion, material aging, longterm effects of load, and the fatigue. The local damage will lead to the destruction of the whole structure, which will result in serious engineering accident. The diagnostic techniques of structural damage have been studied since the 1970s in order to guarantee the structural safety and reduce the economic loss [1]. The aim of structural damage recognition is to find the position and the degree of structural damage, which provides foundation for the followup assessment of structural safety [2]. Damage identification is based on structural vibration, and the basic principle is structural modal parameter (natural frequency, mode shape, etc.) as the function of the structural physical characteristics (mass, damping, and stiffness), and so the change of physical characteristics will cause the change in system dynamic response [3-5].
Another important property which the ideal damage identification method should have is to be able to distinguish the differences of the two deviations caused by structural modeling error and structural damage. How to explain the structural security status and damage degrees by virtue of the information from measurements is still a scientific theory which is to be improved. The structural damage identification method based on the changes of vibration characteristics has been adopted to research for decades. Because the structural vibration modal parameters (such as frequency, mode shapes, and modal damping) are the functions of structural physical parameters (such as mass, stiffness, and damping), changes in the structural physical parameters will inevitably lead to change in structural vibration modal parameters, which is the basic principle of structural damage identification. Damage identification is usually divided into three levels: to judge the occurrence of damage; to determine the location of damage; to solve the extent of damage [6-9]. The early damage identification method generally determines the occurrence of damage by the changes of the frequency before and after the damage. Later, it has been gradually developed by using various modal testing information (such as displacement mode, 
strain mode, and frequency response function) for accurate damage positioning and measurement [10]. The structural damage identification techniques have been combined with modern modal measurement and modern numerical analysis method, and they are playing an important role in the field of civil engineering [11-14].

In terms of algorithms, it usually takes optimization $[15,16]$ or intelligent algorithm [17-19] and other methods to determine the degree of structural damage. Uncertainty widely exists in practical engineering, and the theory and algorithm of uncertain optimization research is significant for the system. In the optimization method of uncertainty, many studies have been done for the uncertainty analysis and solution strategy [20-23]. Due to the fact that there is a lot of uncertain information during the research process of geotechnical engineering, it is difficult for deterministic models to conclude the complicated mechanical property of geotechnical engineering. Data [24] develops a variety of nondeterministic methods on the basis of deterministic back analysis. As the neural network can reflect any nonlinear systems without knowing the nonlinear physical properties of systems, nonlinear dynamical systems of nonparametric research based on the neural network are increasingly developing [25-28]. Data [29] introduces the principle of SDLV and puts forward the precise SDLV damage localization method based on the success of rod damage identification. As for the unreliable results of structural damage identification caused by large and complex structures and a serious shortage of measurement information, data [30] comes up with damage identification methods of partial main frequency substructure. There are noises in both structural model and measurement response which lead to the numerical instability of structural damage identification. Tikhonov regularization method is a common method to improve ill-conditioned matrix. By introducing a smooth function to Tikhonov penalty function, data [31] improves the impact of noise on structural damage. It is an effective way to study the effects of noise on structural damage identification by means of probability. Data [32] presents the information fusion techniques based on Bayesian theory, which is used to improve the accuracy of structural damage identification results. Structural monitoring can only monitor partial measuring points, while the random damage locating vector method can produce better recognition results for truss bridges and steel frame structures [33]. For the problems of inaccurate damage identification of symmetric structure, data [34] proposes the theory of mobile additional mass to change symmetry of the structure.

Physical parameters identification is one of the main research contents of structural health monitoring. According to the change of physical parameters, especially the stiffness, we are able to identify the structure damage, as well as the damage degree and location. In this paper, the natural excitation technique and the extended Kalman filter algorithm are used in shear structure by adopting time domain identification method, and a new method of physical parameter identification based on environmental excitation is put forward to identify the interlaminar stiffness of the shear structure. Numerical simulation results show that the proposed method can well identify structural parameters. With the increase of noise level, convergence time of identified value to the true value elongates and error increases gradually but within the acceptable scope of the project, which shows algorithm has certain robustness to noise.

\section{EKF Principle of Structural Stiffness Identification}

The structural equation of motion under seismic excitations can be expressed as

$$
\mathbf{M} \ddot{\mathbf{x}}+\mathbf{C} \dot{\mathbf{x}}+\mathbf{K}(\boldsymbol{\theta}) \mathbf{x}=-\mathbf{M} \ddot{\mathbf{x}}_{g},
$$

$\mathbf{M}, \mathbf{C}$, and $\mathbf{K}(\boldsymbol{\theta})$ represent mass matrix of $n \times n$ dimensional, damping matrix, and stiffness matrix. Damping matrix $\mathbf{C}$ uses Rayleigh damping; $\ddot{\mathbf{x}}, \dot{\mathbf{x}}$, and $\mathbf{x}$ are the acceleration of the structure, speed, and displacement response; $\ddot{\mathbf{x}}_{g}$ is ground motion acceleration; $n$ is the degree of structure freedom; $\boldsymbol{\theta}$ is structural stiffness parameters to be identified, which dimension is $m$.

At the time of $k$ and $k+1$,

$$
\begin{gathered}
\mathbf{M} \ddot{\mathbf{x}}_{k}+\mathbf{C} \dot{\mathbf{x}}_{k}+\mathbf{K}(\boldsymbol{\theta}) \mathbf{x}_{k}=\mathbf{F}_{k}, \\
\mathbf{M} \ddot{\mathbf{x}}_{k+1}+\mathbf{C} \dot{\mathbf{x}}_{k+1}+\mathbf{K}(\boldsymbol{\theta}) \mathbf{x}_{k+1}=\mathbf{F}_{k+1} .
\end{gathered}
$$

According to Newmark- $\beta$ method, at the time of $k+1$, velocity and displacement can be expressed as

$$
\begin{gathered}
\dot{\mathbf{x}}_{k+1}=\dot{\mathbf{x}}_{k}+\frac{\Delta t}{2}\left(\ddot{\mathbf{x}}_{k}+\ddot{\mathbf{x}}_{k+1}\right), \\
\mathbf{x}_{k+1}=\mathbf{x}_{k}+\dot{\mathbf{x}}_{k} \Delta t+\frac{\Delta t^{2}}{4}\left(\ddot{\mathbf{x}}_{k}+\ddot{\mathbf{x}}_{k+1}\right),
\end{gathered}
$$

$\Delta t$ is discrete time interval.

Substituting (4) into (3) and deducing $\ddot{\mathbf{x}}_{k+1}$, we can deduce the following:

$$
\begin{aligned}
\ddot{\mathbf{x}}_{k+1}= & \mathbf{A}_{1}\left[\mathbf{F}_{k+1}-\mathbf{K}(\boldsymbol{\theta}) \mathbf{x}_{k}-\mathbf{A}_{2} \dot{\mathbf{x}}_{k}-\mathbf{A}_{3} \ddot{\mathbf{x}}_{k}\right], \\
\mathbf{A}_{1} & =\left(\frac{\Delta t^{2}}{4} \mathbf{K}(\boldsymbol{\theta})+\frac{\Delta t}{2} \mathbf{C}+\mathbf{M}\right)^{-1}, \\
\mathbf{A}_{2} & =(\Delta t \mathbf{K}(\boldsymbol{\theta})+\mathbf{C}), \\
\mathbf{A}_{3} & =\left(\frac{\Delta t^{2}}{4} \mathbf{K}(\boldsymbol{\theta})+\frac{\Delta t}{2} \mathbf{C}\right),
\end{aligned}
$$

$\ddot{\mathbf{x}}_{k}$ can be obtained through equation (2) as follows:

$$
\ddot{\mathbf{x}}_{k}=\mathbf{M}^{-1} \mathbf{F}_{k}-\mathbf{M}^{-1} \mathbf{K}(\boldsymbol{\theta}) \mathbf{x}_{k}-\mathbf{M}^{-1} \mathbf{C} \dot{\mathbf{x}}_{k} .
$$

Combining (5) and (7), we can deduce the following:

$$
\begin{aligned}
\left(\ddot{\mathbf{x}}_{k+1}+\ddot{\mathbf{x}}_{k}\right)= & \mathbf{A}_{1} \mathbf{F}_{k+1}-\mathbf{A}_{1} \mathbf{K}(\boldsymbol{\theta}) \mathbf{x}_{k}-\mathbf{A}_{1} \mathbf{A}_{2} \dot{\mathbf{x}}_{k} \\
& -\left(\mathbf{A}_{1} \mathbf{A}_{3}-\mathbf{I}\right) \ddot{\mathbf{x}}_{k} \\
= & \mathbf{a}_{k}^{f}+\mathbf{a}_{k}^{d} \mathbf{x}_{k}+\mathbf{a}_{k}^{v} \dot{\mathbf{x}}_{k},
\end{aligned}
$$




$$
\begin{aligned}
& \mathbf{a}_{k}^{f}=\mathbf{A}_{1} \mathbf{F}_{k+1}-\left[\mathbf{A}_{1} \mathbf{A}_{3}-\mathbf{I}\right] \mathbf{M}^{-1} \mathbf{F}_{k}, \\
& \mathbf{a}_{k}^{d}=-\mathbf{A}_{1} \mathbf{K}(\boldsymbol{\theta})+\left[\mathbf{A}_{1} \mathbf{A}_{3}-\mathbf{I}\right] \mathbf{M}^{-1} \mathbf{K}(\boldsymbol{\theta}), \\
& \mathbf{a}_{k}^{v}=-\mathbf{A}_{1} \mathbf{A}_{2}+\left[\mathbf{A}_{1} \mathbf{A}_{3}-\mathbf{I}\right] \mathbf{M}^{-1} \mathbf{C} .
\end{aligned}
$$

Substituting (8) into (4), we can deduce the following formula:

$$
\begin{aligned}
\dot{\mathbf{x}}_{k+1} & =\dot{\mathbf{x}}_{k}+\frac{\Delta t}{2}\left(\mathbf{a}_{k}^{f}+\mathbf{a}_{k}^{d} \mathbf{x}_{k}+\mathbf{a}_{k}^{v} \dot{\mathbf{x}}_{k}\right) \\
& =\frac{\Delta t}{2} \mathbf{a}_{k}^{f}+\frac{\Delta t}{2} \mathbf{a}_{k}^{d} \mathbf{x}_{k}+\left(\frac{\Delta t}{2} \mathbf{a}_{k}^{v}+\mathbf{I}\right) \dot{\mathbf{x}}_{k}, \\
\mathbf{x}_{k+1} & =\mathbf{x}_{k}+\dot{\mathbf{x}}_{k} \Delta t+\frac{\Delta t^{2}}{4}\left(\mathbf{a}_{k}^{f}+\mathbf{a}_{k}^{d} \mathbf{x}_{k}+\mathbf{a}_{k}^{v} \dot{\mathbf{x}}_{k}\right) \\
& =\frac{\Delta t^{2}}{4} \mathbf{a}_{k}^{f}+\left(\frac{\Delta t^{2}}{4} \mathbf{a}_{k}^{d}+\mathbf{I}\right) \mathbf{x}_{k}+\left(\frac{\Delta t^{2}}{4} \mathbf{a}_{k}^{v}+\Delta t \mathbf{I}\right) \dot{\mathbf{x}}_{k} .
\end{aligned}
$$

Write formula (10) in the matrix form

$$
\begin{aligned}
\left\{\begin{array}{c}
\mathbf{x}_{k+1} \\
\dot{\mathbf{x}}_{k+1}
\end{array}\right\}= & {\left[\begin{array}{cc}
\left(\frac{\Delta t^{2}}{4} \mathbf{a}_{k}^{d}+\mathbf{I}\right) & \left(\frac{\Delta t^{2}}{4} \mathbf{a}_{k}^{v}+\Delta t \mathbf{I}\right) \\
\frac{\Delta t}{2} \mathbf{a}_{k}^{d} & \left(\frac{\Delta t}{2} \mathbf{a}_{k}^{v}+\mathbf{I}\right)
\end{array}\right]\left\{\begin{array}{l}
\mathbf{x}_{k} \\
\dot{\mathbf{x}}_{k}
\end{array}\right\} } \\
& +\left\{\begin{array}{c}
\frac{\Delta t^{2}}{4} \mathbf{I} \\
\frac{\Delta t}{2} \mathbf{I}
\end{array}\right\} \mathbf{a}_{k}^{f},
\end{aligned}
$$

I is the unit matrix of $n \times n$.

Using the method of Newmark- $\beta$, we can transform formula (1) into discrete equation (11).

Let

$$
\mathbf{y}_{k}=\left\{\begin{array}{lll}
\mathbf{x}_{k} & \dot{\mathbf{x}}_{k} & \boldsymbol{\theta}_{k}
\end{array}\right\}^{T} .
$$

Then structural equation of state can be rewritten as

$$
\begin{aligned}
\mathbf{y}_{k+1}= & {\left[\begin{array}{ccc}
\left(\frac{\Delta t^{2}}{4} \mathbf{a}_{k}^{d}(\boldsymbol{\theta})+\mathbf{I}\right) & \left(\frac{\Delta t^{2}}{4} \mathbf{a}_{k}^{v}(\boldsymbol{\theta})+\Delta t \mathbf{I}\right) & \mathbf{0}_{n \times m} \\
\frac{\Delta t}{2} \mathbf{a}_{k}^{d}(\boldsymbol{\theta}) & \left(\frac{\Delta t}{2} \mathbf{a}_{k}^{v}(\boldsymbol{\theta})+\mathbf{I}\right) & \mathbf{0}_{n \times m} \\
\mathbf{0}_{m \times n} & \mathbf{0}_{m \times n} & \mathbf{0}_{m \times m}
\end{array}\right] \mathbf{y}_{k} } \\
& +\left\{\begin{array}{c}
\frac{\Delta t^{2}}{4} \mathbf{I} \\
\frac{\Delta t}{2} \mathbf{I} \\
\mathbf{0}_{m \times n}
\end{array}\right\} \mathbf{a}_{k}^{f} .
\end{aligned}
$$

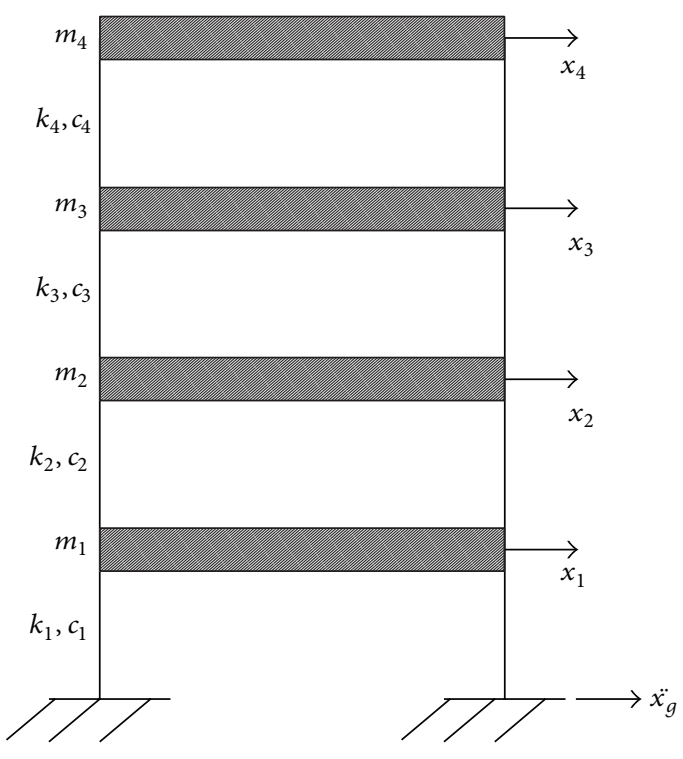

FIGURE 1: Structure diagram.

TABLE 1: Structural parameters.

\begin{tabular}{lcccc}
\hline$i$ & 1 & 2 & 3 & 4 \\
\hline$m_{i}(\mathrm{~kg})$ & 900 & 675 & 675 & 450 \\
$k_{i}\left(\mathrm{Nm}^{-1}\right)$ & 12000 & 11000 & 10000 & 9000 \\
\hline
\end{tabular}

Let

$$
\begin{aligned}
& \boldsymbol{\Phi}_{k}=\left[\begin{array}{ccc}
\left(\frac{\Delta t^{2}}{4} \mathbf{a}_{k}^{d}(\boldsymbol{\theta})+\mathbf{I}\right) & \left(\frac{\Delta t^{2}}{4} \mathbf{a}_{k}^{v}(\boldsymbol{\theta})+\Delta t \mathbf{I}\right) & \mathbf{0}_{n \times m} \\
\frac{\Delta t}{2} \mathbf{a}_{k}^{d}(\boldsymbol{\theta}) & \left(\frac{\Delta t}{2} \mathbf{a}_{k}^{v}(\boldsymbol{\theta})+\mathbf{I}\right) & \mathbf{0}_{n \times m} \\
\mathbf{0}_{m \times n} & \mathbf{0}_{m \times n} & \mathbf{0}_{m \times m}
\end{array}\right], \\
& \boldsymbol{\Gamma}_{k}=\left\{\begin{array}{lll}
\frac{\Delta t^{2}}{4} \mathbf{I} & \frac{\Delta t}{2} \mathbf{I} & \mathbf{0}_{m \times n}
\end{array}\right\}^{T} .
\end{aligned}
$$

If the process noise exists in the system, (13) can be rewritten as

$$
\mathbf{y}_{k+1}=\boldsymbol{\Phi}_{k} \mathbf{y}_{k}+\boldsymbol{\Gamma}_{k} \boldsymbol{\alpha}_{k}^{f}+\boldsymbol{\omega}_{k},
$$

$\boldsymbol{\omega}_{k}$ is the process noise of system.

The supplementary system observation equation is

$$
\mathbf{z}_{k+1}=\mathbf{H}_{k} \mathbf{y}_{k}+\boldsymbol{v}_{k},
$$

$\mathbf{H}_{k}$ is the observation matrix of system and $\boldsymbol{v}_{k}$ is the observation noise of the system.

Assuming that process noise and observation noise are independent of each other, the covariance matrix of process noise and observation noise is $\mathbf{N}=\mathbf{0}$. Given the system initial value $\mathbf{y}_{0}$, the initial value of process noise covariance matrix $\mathbf{P}_{0}$, and observation noise covariance matrix $\mathbf{R}$, discrete augmented state vector $\widehat{\mathbf{y}}_{k+1}$ and covariance matrix $\widehat{\mathbf{P}}_{k+1}$ can be estimated according to the extended Kalman filter method. 


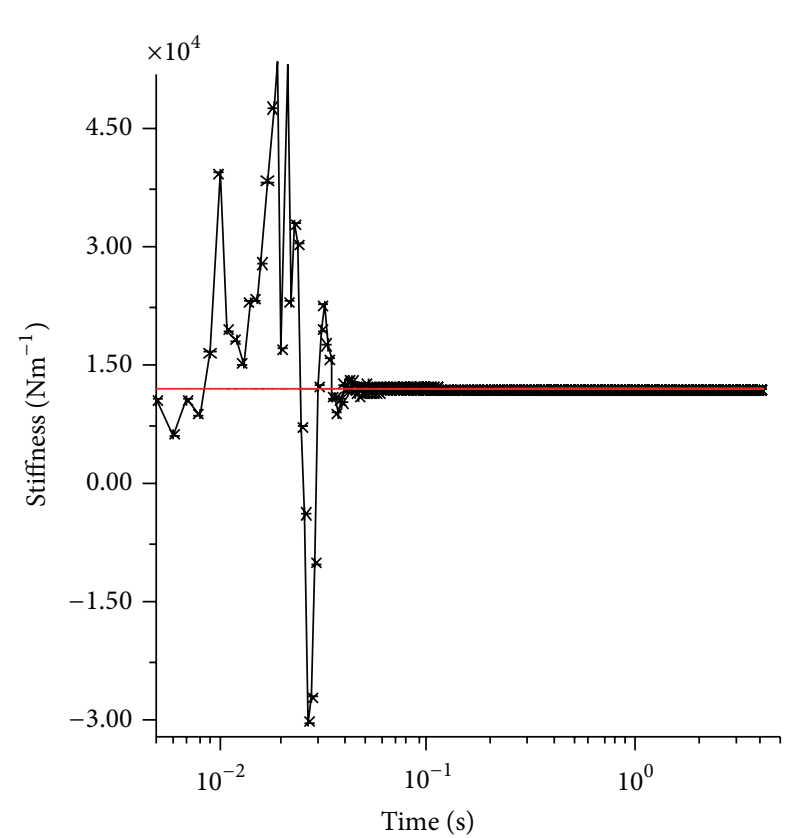

(a) The first layer stiffness

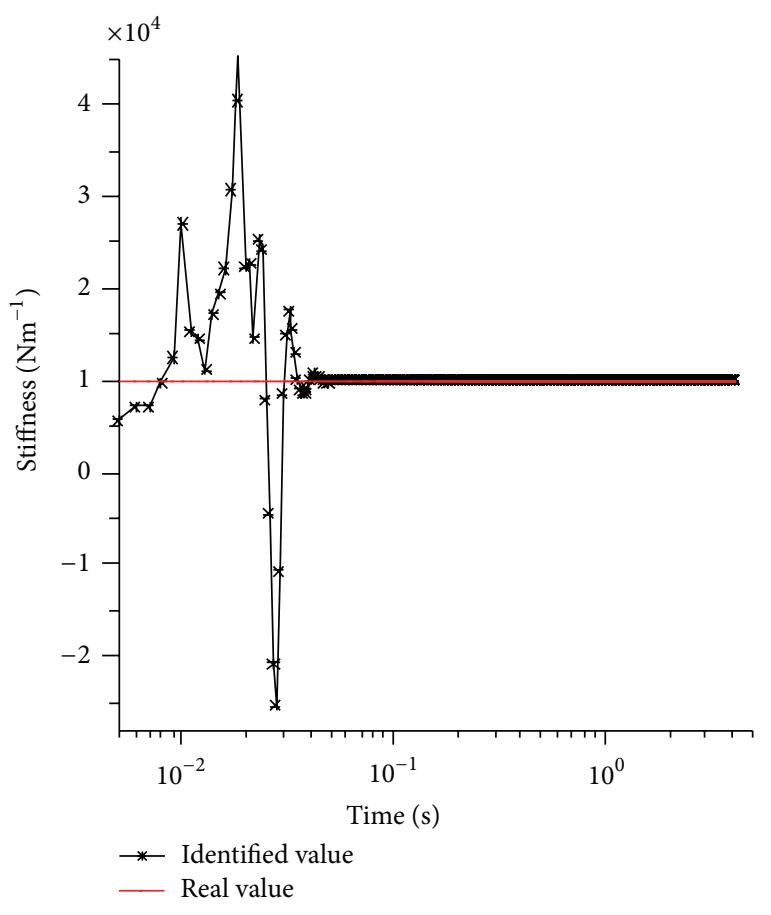

(c) The third layer stiffness

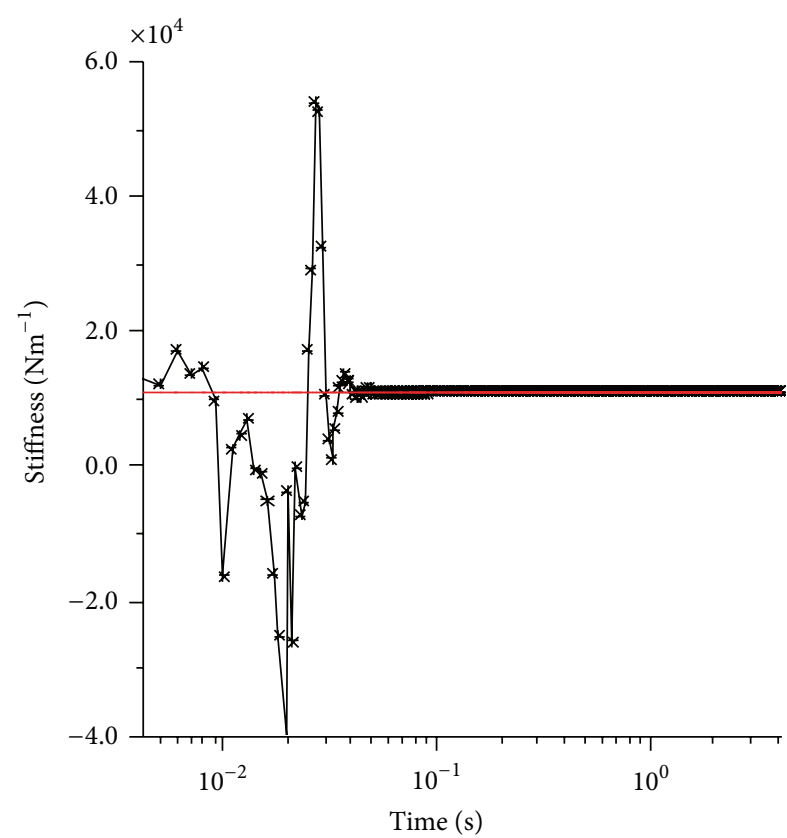

(b) The second layer stiffness

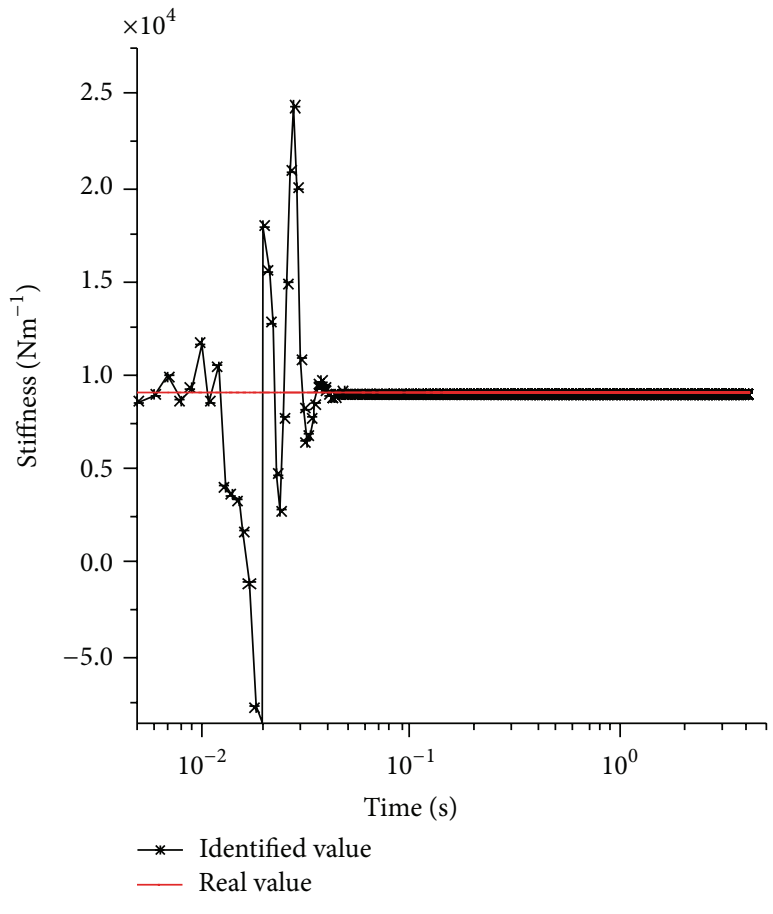

(d) The fourth layer stiffness

FIGURE 2: Structural stiffness identification result without noise.

(1) According to formula (15), calculate and predict the state vector $\widetilde{\mathbf{y}}_{k+1}$.

(2) Calculation of covariance prediction equation is

$$
\widetilde{\mathbf{P}}_{k+1}=\boldsymbol{\Phi}_{k} \mathbf{P}_{k} \boldsymbol{\Phi}_{k}^{T}
$$

(3) The gain matrix is

$$
\mathbf{K}_{k+1}=\widetilde{\mathbf{P}}_{k+1} \mathbf{H}_{k+1}^{T}\left(\mathbf{H}_{k+1} \widetilde{\mathbf{P}}_{k+1} \mathbf{H}_{k+1}^{T}+\mathbf{R}\right)^{-1} \text {. }
$$

(4) State filtering equation is

$$
\widehat{\mathbf{x}}_{k+1}=\widetilde{\mathbf{x}}_{k+1}+\mathbf{K}_{k+1}\left(\mathbf{z}_{k+1}-\mathbf{H}_{k+1} \widetilde{\mathbf{x}}_{k+1}\right) .
$$




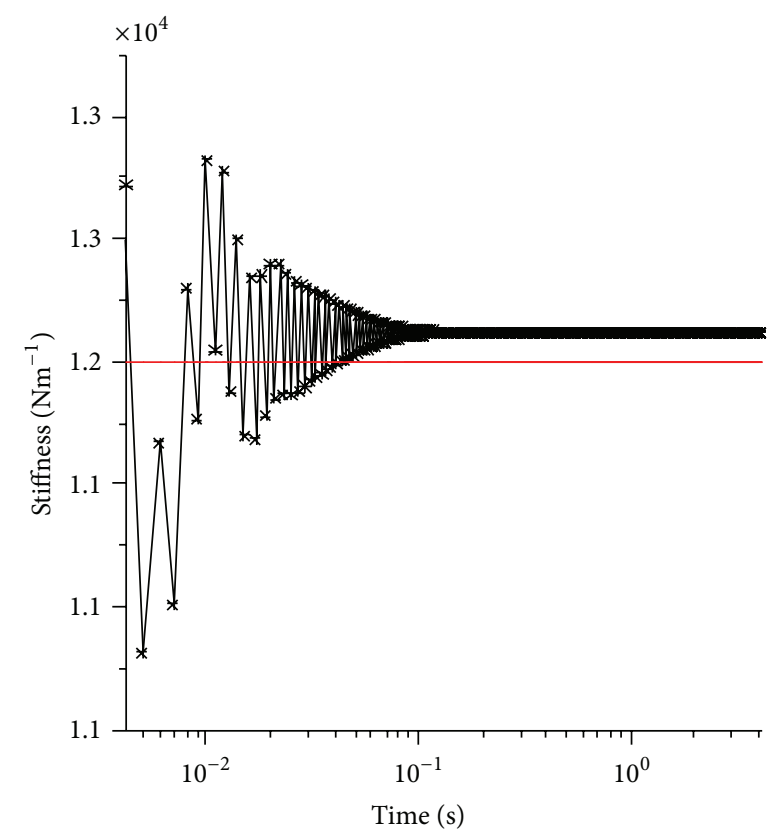

(a) The first layer stiffness

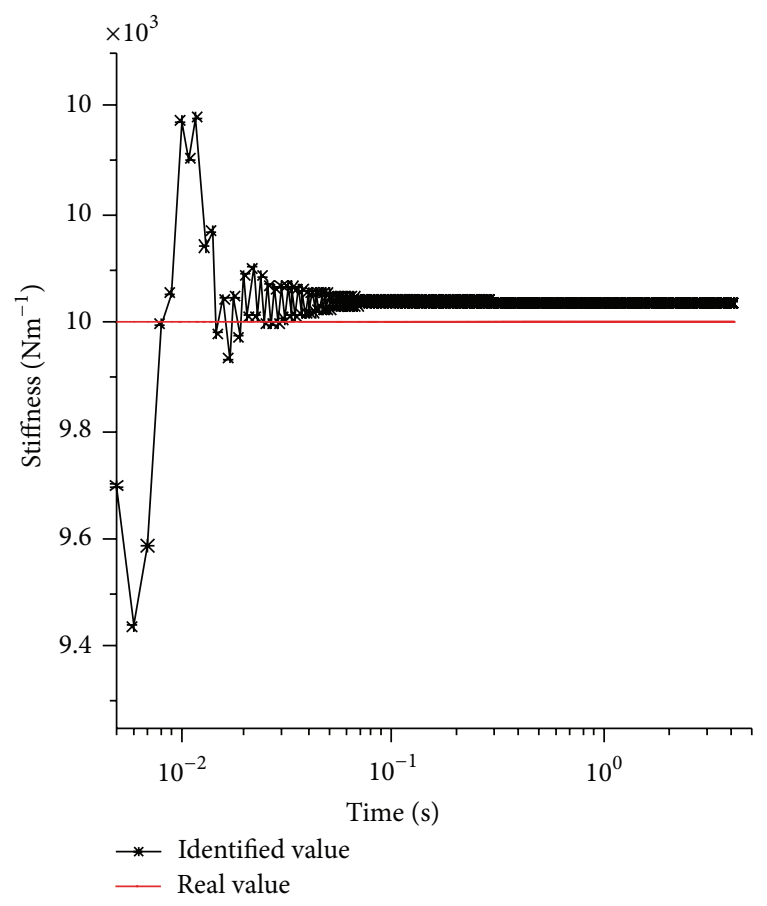

(c) The third layer stiffness

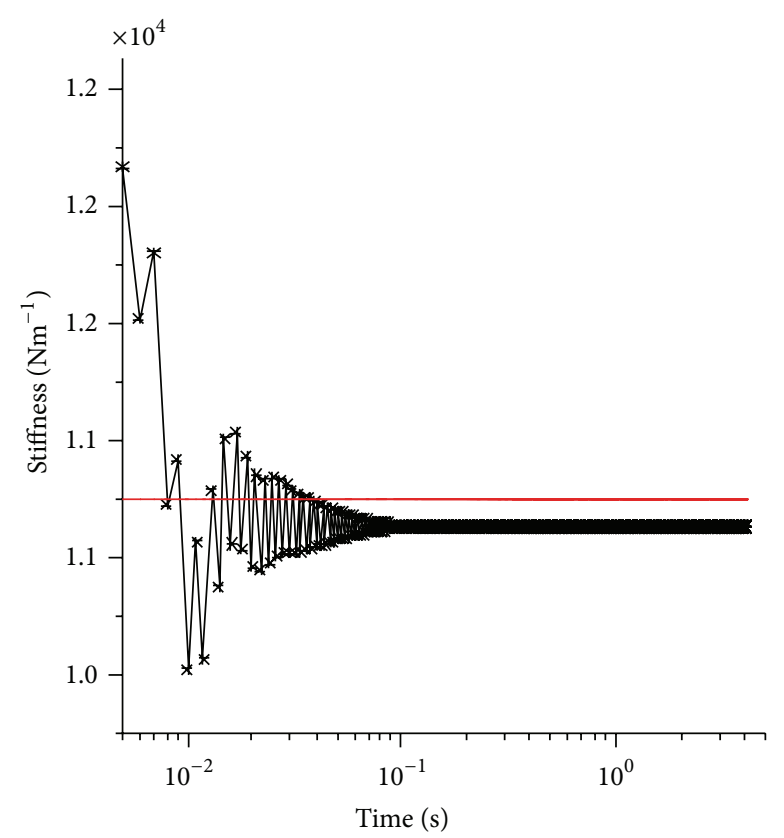

(b) The second layer stiffness

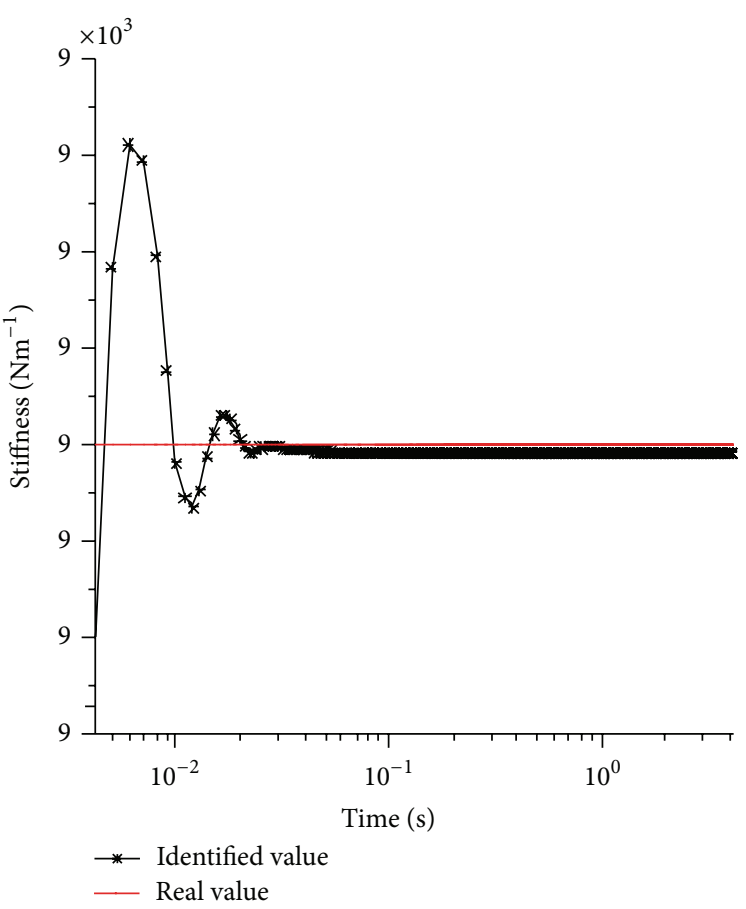

(d) The four layer stiffness

FIGURE 3: Stiffness identification results when SNR is 40.

(5) Error covariance filtering equation is

$$
\mathbf{P}_{k+1}=\widetilde{\mathbf{P}}_{k+1}-\mathbf{K}_{k+1} \mathbf{H}_{k+1} \widetilde{\mathbf{P}}_{k+1} .
$$

\section{The Numerical Simulation}

To validate the effectiveness of the algorithm, a four-story shear structure is considered, as shown in Figure 1. Structure damping is the Rayleigh damping; quality factor is
$6.984 \times 10^{-3}$ and stiffness coefficient is $9.390 \times 10^{-4}$; the rest parameters of the structure are shown in Table 1 . The input of basement is elecentro wave. Use the method of time-history analysis to calculate structural response and gather each layer displacement response as measurements to identify stiffness between the layers.

Three working conditions in this paper are considered, which are measurements without noise, measurements with $1 \%$ of noise, and measurements with $3 \%$ of noise. All the 


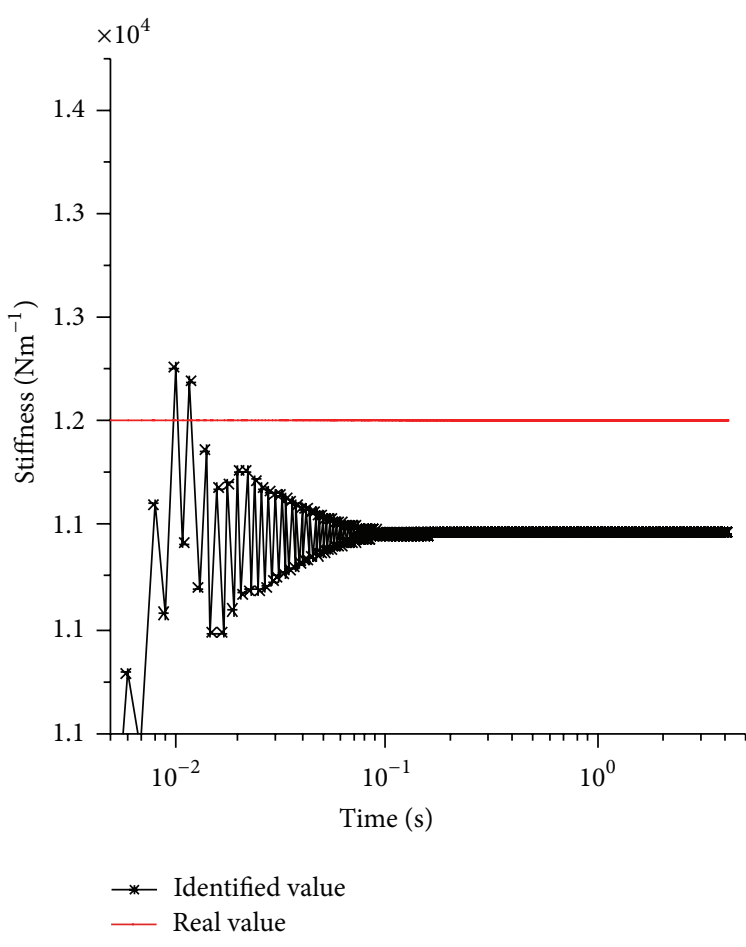

(a) The first layer stiffness

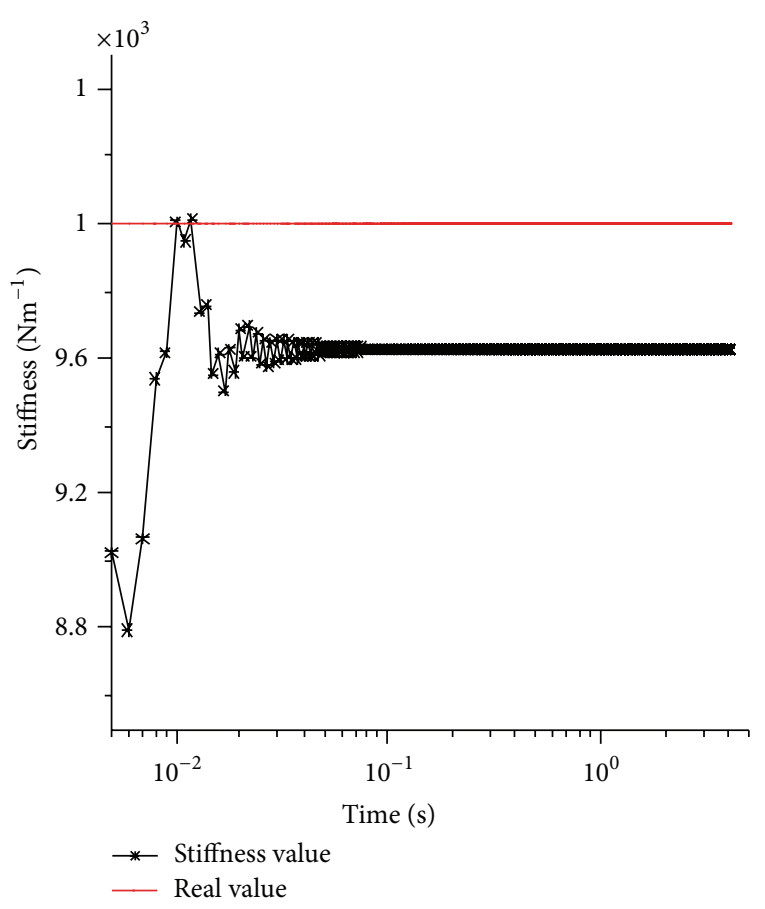

(c) The third layer stiffness

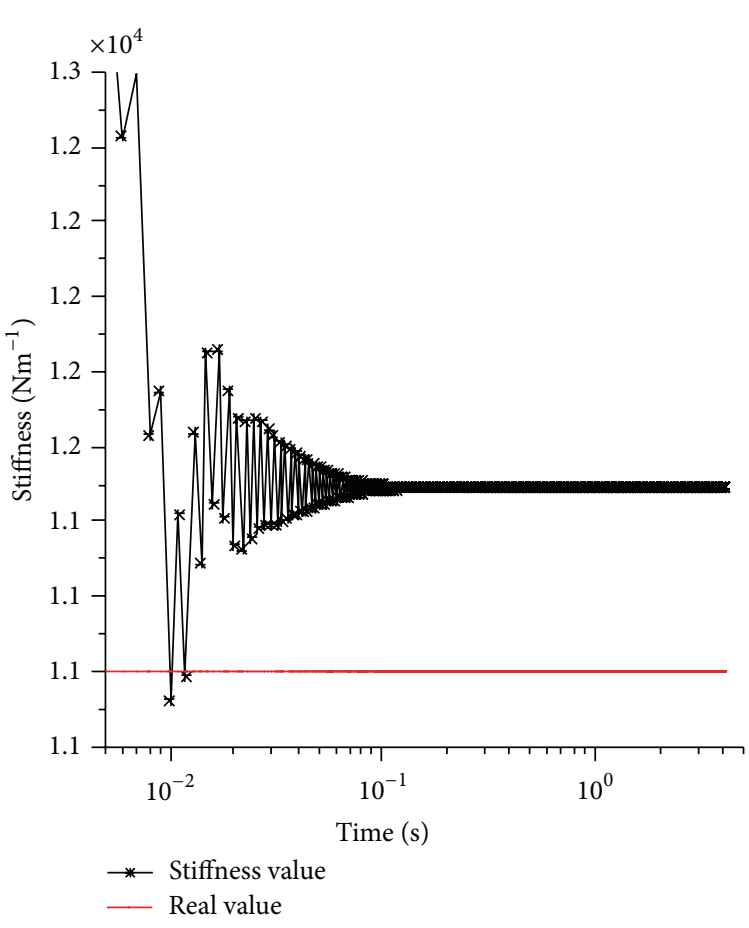

(b) The second layer stiffness

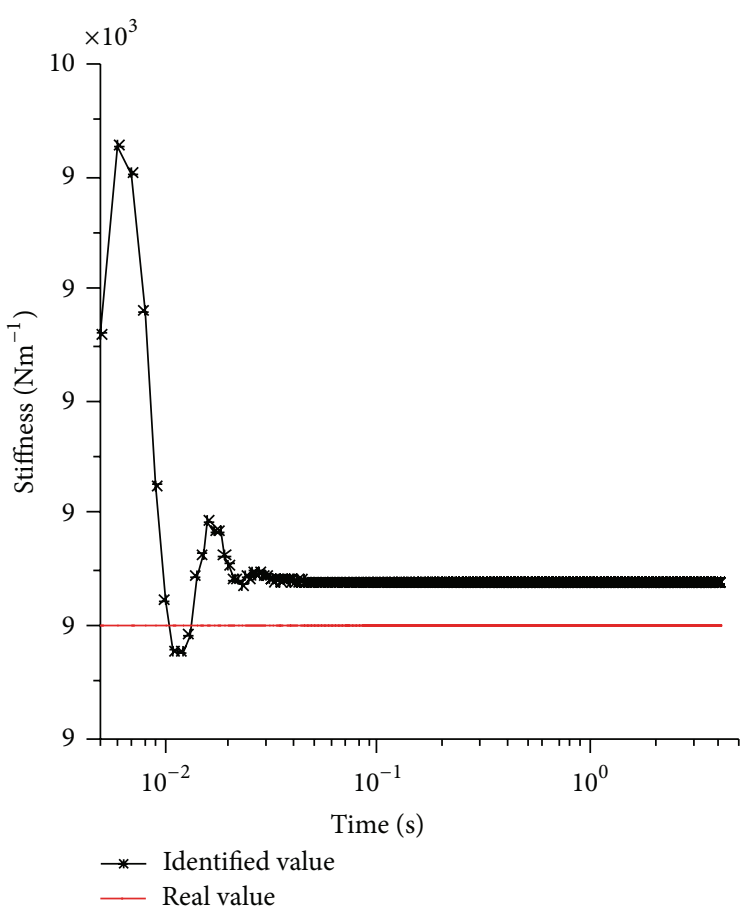

(d) The fourth layer stiffness

FIGURE 4: Stiffness identification results when SNR is 30 .

noises are zero mean white noise. Figure 2 shows the structure stiffness identification value without noise, from which we can see that identified value of stiffness converges to the true value quickly. Figure 3 shows the identification result of measurements with $1 \%$ of noise. Due to the effect of the noise, there is a certain error between identified value and true value. Table 2 shows the error level; the maximum error is within the $1 \%$ with the situation of $1 \%$ of noise. Figure 4 
TABLE 2: Structural stiffness identification results.

\begin{tabular}{|c|c|c|c|c|c|c|}
\hline \multirow{2}{*}{ Stiffness } & \multicolumn{6}{|c|}{ Noise level } \\
\hline & No noise & Error level \% & $\mathrm{SNR}=40$ & Error level \% & $\mathrm{SNR}=30$ & Error level \% \\
\hline$K_{1}$ & 12000 & 0 & 12118 & 0.98 & 11459 & -4.51 \\
\hline$K_{2}$ & 11000 & 0 & 10904 & -0.87 & 11491 & 4.46 \\
\hline$K_{3}$ & 10000 & 0 & 10039 & 0.39 & 9628 & -3.72 \\
\hline$K_{4}$ & 9000 & 0 & 8991 & -0.10 & 9038 & 0.42 \\
\hline
\end{tabular}

shows the measurements with $3 \%$ of noise. From Table 2, we can see that the maximum error is $4.6 \%$, and it is within the acceptable range of the engineering.

\section{Conclusion}

The paper using the method of Newmark- $\beta$ disperses the equations of motion and deduces the state transition equation containing the stiffness parameters to be identified. By the extended Kalman filter algorithm to identify the stiffness parameters, the conclusion can be drown as follows.

(i) Expanding order state equation is nonlinear state equation of state variables, and the state transition matrix is generally through the partial derivative of the nonlinear equation, which solving process is complicated. This paper directly deduces the state transition matrix by Newmark- $\beta$ method, and the result is concise and intuitive.

(ii) Noise affects the identification precision. When there is no noise in acquisition response, the identification stiffness converges to the true stiffness precisely. With the noise increases, the error of identification precision increases too. When the SNR amounts to forty, the error is within $1 \%$, and, when the SAR is thirty, the error is within 5\%. The speed of error increasing surpasses that of noise increasing.

(iii) Noise affects the speed of identification. When there is no noise in the acquisition response, the algorithm convergence accesses the truth value. And, when noise increases, the speed of algorithm convergence becomes slow. In the practical condition that noise has affected acquisition response, the time length of acquisition should be guaranteed so that the algorithm converges to the stable value, which can ensure reliable identification results.

(iv) From the results of numerical simulation, the proposed algorithm has different identification precisions to the various layers of shear structure, which is in the increasing condition from the bottom to the top. The incentive of structure is under the affection of earthquake, and greater response of structural layer can be obtained from the higher ground; the different responses between the layers will affect algorithm identification precision.

\section{Conflict of Interests}

The authors declare that there is no conflict of interests regarding the publication of this paper.

\section{Acknowledgments}

The authors express their special appreciation to all authors of the following references due to their support for the paper and anonymous referee for valuable suggestions on the earlier version of the paper. In addition, this work is supported by NSFC under Grant no. 51174261 and China Shenhua Energy Company Limited (CSIE12021243).

\section{References}

[1] H.-P. Zhu, J. Yu, and J.-B. Zhang, "A summary review and advantages of vibration-based damage identification methods in structural health monitoring," Engineering Mechanics, vol. 28, no. 2, pp. 1-11, 2011.

[2] H. Kim and H. Melhem, "Damage detection of structures by wavelet analysis," Journal of Engineering Structures, vol. 26, pp. $7-14,2004$.

[3] J. P. Ou and G. Y. Wang, Random Vibration of Structures, Press of Higher Education, Beijing, China, 1998.

[4] O. S. Salawu, "Detection of structural damage through changes in frequency: a review," Engineering Structures, vol. 19, no. 9, pp. 718-723, 1997.

[5] F. Vestroni and D. Capecchi, "Damage detection in beam structures based on frequency measurements," Journal of Engineering Mechanics, vol. 126, no. 7, pp. 761-768, 2000.

[6] D. L. Zheng, Z. F. Li, and H. X. Hua, "Summary review of structural initial damage identification methods," Journal of Vibration and Shock, vol. 21, no. 2, pp. 1-6, 2002.

[7] S. Y. Du, X. G. Yin, and H. Chen, "Sieve method for identifying damage based on matrix perturbation theory," Chinese Journal of Applied Mechanics, vol. 25, no. 1, pp. 75-78, 2008.

[8] M. F. Elkordy, K. C. Chang, and G. C. Lee, "Application of neural networks in vibrational signature analysis," Journal of Engineering Mechanics, vol. 120, no. 2, pp. 250-264, 1994.

[9] H.-S. Tang, S.-T. Xue, R. Chen, and K. Jin, "Sequential LSSVM for structural systems identification," Journal of Vibration Engineering, vol. 19, no. 3, pp. 382-387, 2006.

[10] S. P. He and J. Song, "Unbiased estimation of Markov jump systems with distributed delays," Signal Processing, vol. 100, pp. 85-92, 2014.

[11] S. P. He and F. Liu, "Robust finite-time estimation of Markovian jumping systems with bounded transition probabilities," Applied Mathematics and Computation, vol. 222, pp. 297-306, 2013. 
[12] S. P. He, "Resilient $L_{2}-L_{\infty}$ filtering of uncertain Markovian jumping systems within the finite-time interval," Abstract and Applied Analysis, vol. 2013, Article ID 791296, 7 pages, 2013.

[13] S. P. He and F. Liu, "Robust peak-to-peak filtering for Markov jump systems," Signal Processing, vol. 90, no. 2, pp. 513-522, 2010.

[14] S. P. He and F. Liu, "Adaptive observer-based fault estimation for stochastic Markovian jumping systems," Abstract and Applied Analysis, vol. 2012, Article ID 176419, 11 pages, 2012.

[15] A. K. Pandey, M. Biswas, and M. M. Samman, "Damage detection from changes in curvature mode shapes," Journal of Sound and Vibration, vol. 145, no. 2, pp. 321-332, 1991.

[16] W.-X. Ren and G. D. Roeck, "Structural damage identification using modal data. I: simulation verification," Journal of Structural Engineering, vol. 128, no. 1, pp. 87-95, 2002.

[17] J. G. Sun, "Least-squares solutions of a class of inverse eigenvalue problems," Mathematica Numerica Sinica, vol. 9, no. 2, pp. 206-216, 1987.

[18] M. F. Elkordy, K. C. Chang, and G. C. Lee, "Application of neural networks in vibrational signature analysis," Journal of Engineering Mechanics, vol. 120, no. 2, pp. 250-265, 1994.

[19] H.-S. Tang, S.-T. Xue, R. Chen, and K. Jin, "Sequential LSSVM for structural systems identification," Journal of Vibration Engineering, vol. 19, no. 3, pp. 382-387, 2006.

[20] B. Liu, "Uncertain set theory and uncertain inference rule with application to uncertain control," Journal of Uncertain Systems, vol. 4, pp. 83-98, 2010.

[21] B. Liu, "Some research problems in uncertainty theory," Journal of Uncertain Systems, vol. 3, pp. 3-10, 2009.

[22] G. D. Tian, M. C. Zhou, and J. W. Chu, "A chance constrained programming approach to determine the optimal disassembly sequence," IEEE Transactions on Automation Science and Engineering, vol. 10, pp. 1004-1013, 2013.

[23] G. Tian, M. Zhou, J. Chu, and Y. Liu, "Probability evaluation models of product disassembly cost subject to random removal time and different removal labor cost," IEEE Transactions on Automation Science and Engineering, vol. 9, no. 2, pp. 288-295, 2012.

[24] B. S. Zhou and W. S. Zhu, "Study on forecasting the parameters of roadway surrounding rock based by artificial neural networks," Rock and Soil Mechanics, vol. 20, no. 1, pp. 22-26, 1999.

[25] S. F. Masri, J. P. Caffrey, T. K. Caughey, A. W. Smyth, and A. G. Chassiakos, "Identification of the state equation in complex non-linear systems," International Journal of NonLinear Mechanics, vol. 39, no. 7, pp. 1111-1127, 2004.

[26] H. M. Chen, G. Z. Qi, J. C. S. Yang, and F. Amini, "Neural network for structural dynamic model identification," Journal of Engineering Mechanics, vol. 121, no. 12, pp. 1377-1381, 1995.

[27] M. Nakaimra, S. F. Masri, and T. K. Caughey, "A method for non parametric: damage detection through the use of neural networks," Earthquake Engineering \& Structural Dynamics, vol. 27, pp. 997-1010, 1998.

[28] S. F. Masri, N. F. Hunter, and A. G. Chassiakos, "Application of neural networks for detection of nonlinear systems," Engineering Mechanics, vol. 126, pp. 666-676, 2000.

[29] Y.-H. An and J.-P. Ou, "Experimental and numerical studies on precise damage localization of a long-span steel truss bridge model," Chinese Journal of Computational Mechanics, vol. 28, no. 5, pp. 730-736, 2011.
[30] J. L. Hou, J. P. Ou, and L. Jankowski, “The study and experiment of substructure damage identification based on local primary frequency," Engineering Mechanics, vol. 29, pp. 99-105, 2012.

[31] C. Zhang, G.-Q. Song, and G.-Y. Wu, "Structure damage identification by finite element model updated with improved Tikhonov regularization," Engineering Mechanics, vol. 29, no. 2, pp. 29-44, 2012.

[32] X. D. Sun, J. P. Ou, G. L. Hou et al., "Damage Identification based on Bayesian fusion of global and local information," Engineering Mechanics, vol. 29, pp. 234-239, 2012.

[33] Q. P. Wang and X. Guo, "DLV-based research on damage identification of steel frame," World Earthquake Engineering, vol. 28, pp. 50-55, 2012.

[34] C. Deng, P.-Y. Gu, and L. Tang, "Damage diagnosis based on natural frequencies of a structure by adding known masses," Journal of Vibration and Shock, vol. 29, no. 7, pp. 135-138, 2010. 


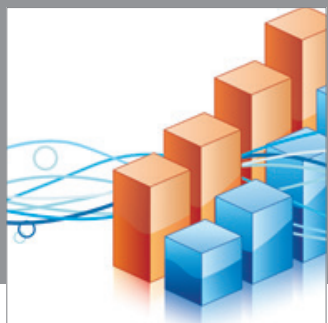

Advances in

Operations Research

mansans

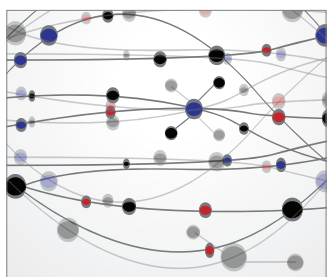

The Scientific World Journal
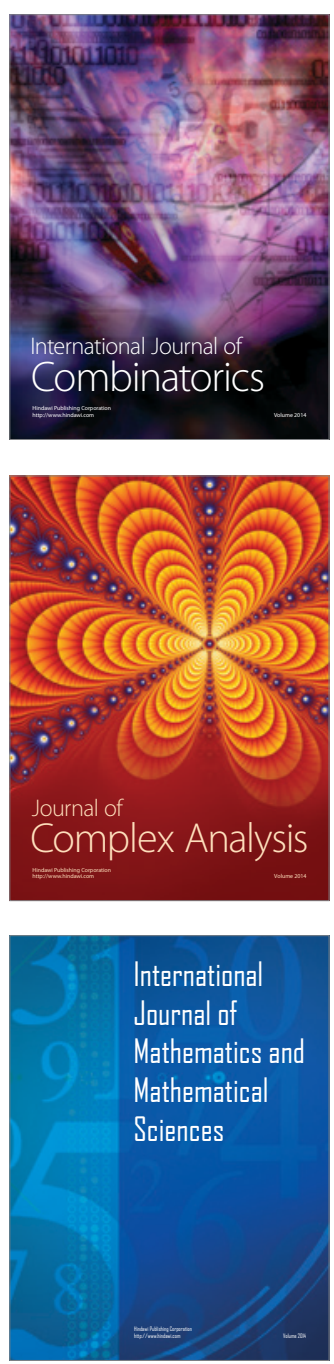
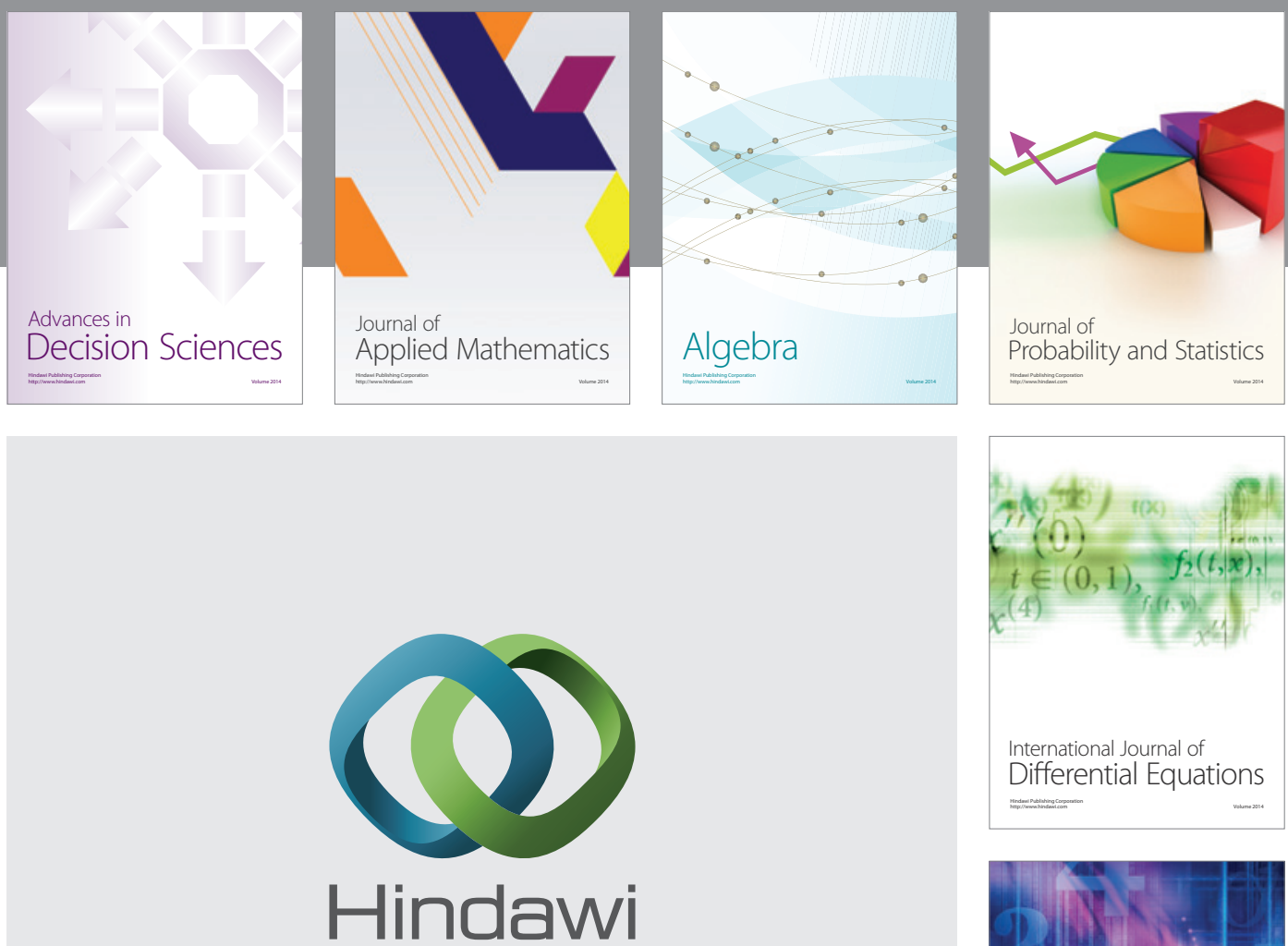

Submit your manuscripts at http://www.hindawi.com
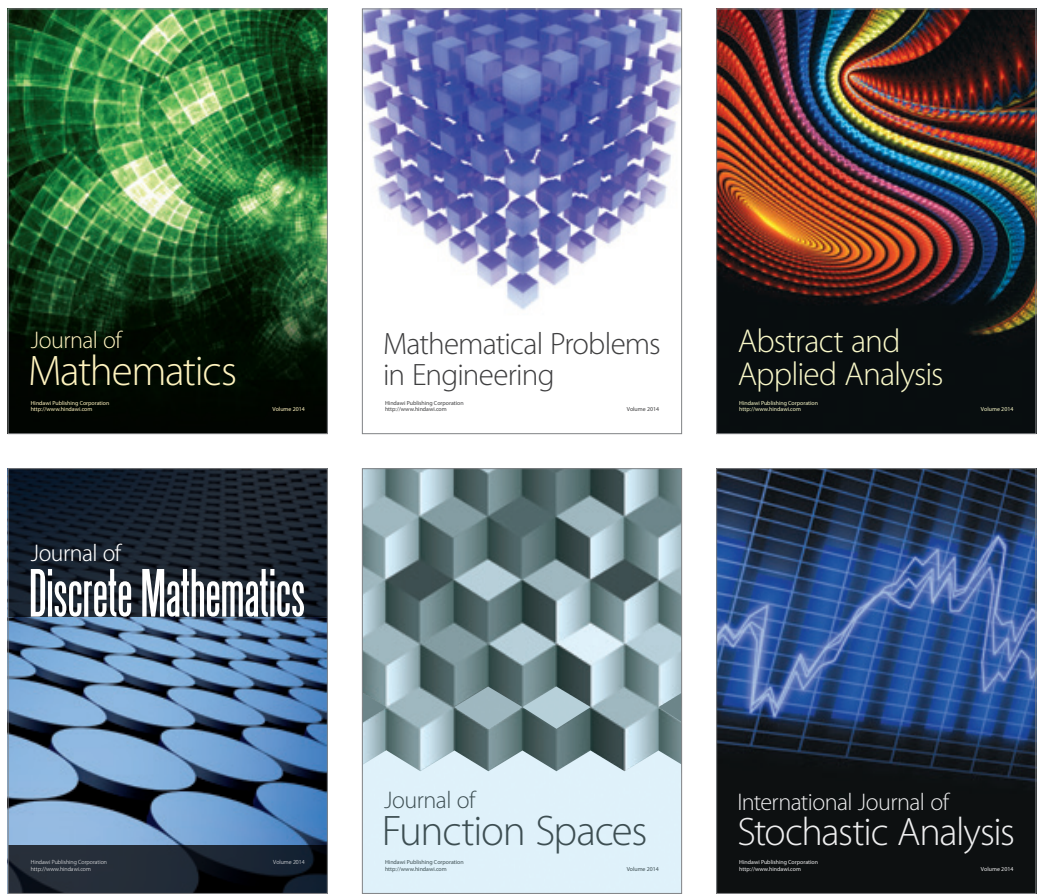

Journal of

Function Spaces

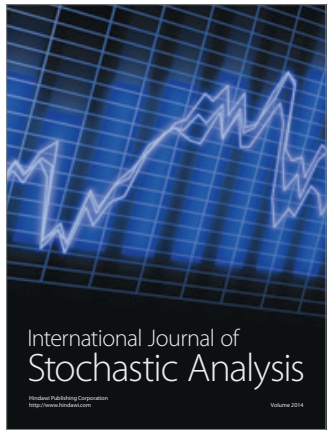

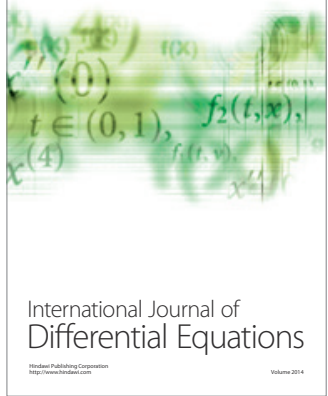
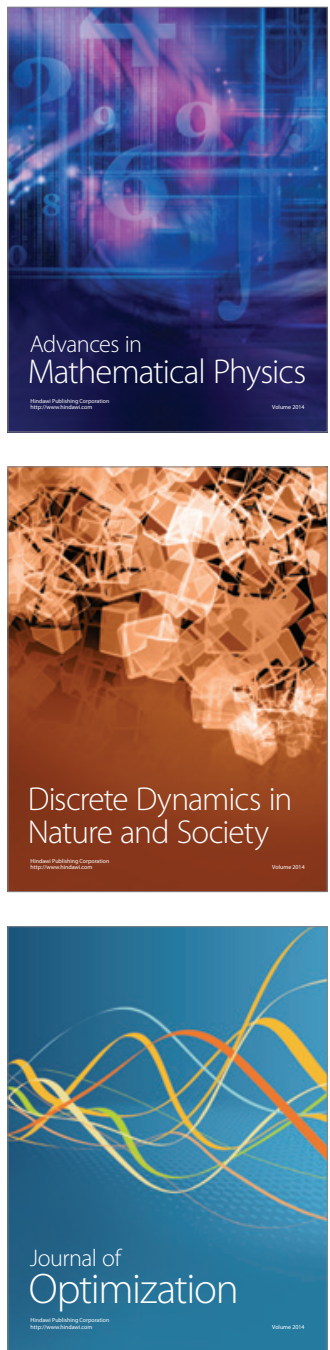